
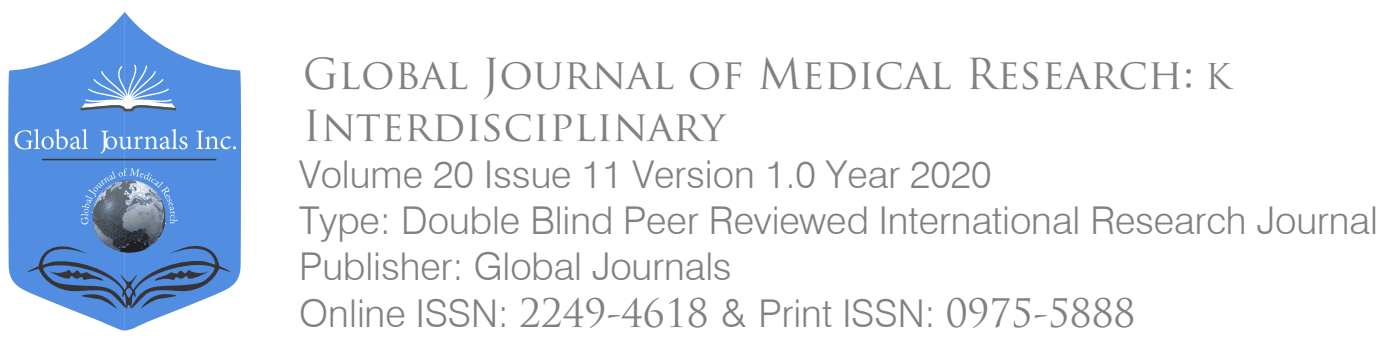

\title{
Balance and Fall Risk Outcome Measures for Patients 65 Years or Older in the Outpatient Physical Therapy Setting: What are the Current Trends?
}

By Debra R. McDowell, PT, PhD, Denise Gobert, PT, MEd. PhD, Tate Jarzombek, DPT, Taylor Koudela, DPT, Katelynn McGrath, DPT \& Rachel Raulerson, DPT

Texas State University

Abstract-Background \& Objectives: Occurrence of falls in older adults contributes to increased morbidity and mortality, exponential healthcare costs, and reduced quality of life. Utilization of outcome measures for screening and assessing fall risk in older patients contributes to reduced fall-related injuries, deaths, and healthcare costs, while promoting improved mobility and health status. The purpose of this study was to explore the utilization of outcome measures assessing balance and fall risk in patients 65 years and up within the outpatient physical therapy setting. Secondary aims included determining the most frequently used outcome measures as well as examining any associations between demographic variables and clinicians' screening habits.

Keywords: older adults, geriatrics, falls, balance, outcome measures, screening, physical therapy practice.

GJMR-K Classification: NLMC Code: WB 460



Strictly as per the compliance and regulations of:

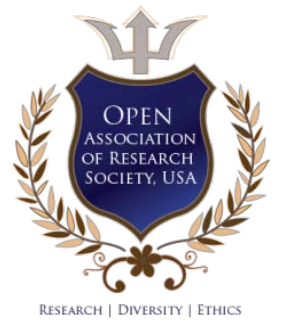

(c) 2020. Debra R. McDowell, PT, PhD, Denise Gobert, PT, MEd. PhD, Tate Jarzombek, DPT, Taylor Koudela, DPT, Katelynn McGrath, DPT \& Rachel Raulerson, DPT. This is a research/review paper, distributed under the terms of the Creative Commons Attribution-Noncommercial 3.0 Unported License http://creativecommons.org/licenses/by-nc/3.0/), permitting all non-commercial use, distribution, and reproduction in any medium, provided the original work is properly cited. 


\title{
Balance and Fall Risk Outcome Measures for Patients 65 Years or Older in the Outpatient Physical Therapy Setting: What are the Current Trends?
}

\author{
Debra R. McDowell, PT, PhD ${ }^{\alpha}$, Denise Gobert, PT, MEd. PhD ${ }^{\sigma}$, Tate Jarzombek, DPT ${ }^{\rho}$, \\ Taylor Koudela, DPT ${ }^{\omega}$, Katelynn McGrath, DPT ${ }^{\ddagger} \&$ Rachel Raulerson, DPT ${ }^{\S}$
}

Abstract- Background \& Objectives: Occurrence of falls in older adults contributes to increased morbidity and mortality, exponential healthcare costs, and reduced quality of life. Utilization of outcome measures for screening and assessing fall risk in older patients contributes to reduced fall-related injuries, deaths, and healthcare costs, while promoting improved mobility and health status. The purpose of this study was to explore the utilization of outcome measures assessing balance and fall risk in patients 65 years and up within the outpatient physical therapy setting. Secondary aims included determining the most frequently used outcome measures as well as examining any associations between demographic variables and clinicians' screening habits.

Methods and Measures: Physical therapists and physical therapist assistants were contacted via email with a link to an online survey that included questions regarding demographic data, physical therapy practice, and utilization of outcome measures for balance and fall risk. Frequency distributions were generated to illustrate demographic variables and Pearson Chi-squared analysis was used to explore associations between demographic variables and the utilization of outcome measures to screen balance in patients 65 years and older.

Keywords: older adults, geriatrics, falls, balance, outcome measures, screening, physical therapy practice.

Abstract-Background and Objectives: Occurrence of falls in older adults contributes to increased morbidity and mortality, exponential healthcare costs, and reduced quality of life. Utilization of outcome measures for screening and assessing fall risk in older patients for implementation of plan of care contributes to reduced fall-related injuries, deaths, and healthcare costs, while promoting improved mobility, function, and health status. These outcome measures are described and recommended by the American Geriatric Society, American Physical Therapy Association, Academy of Neurologic Physical Therapy, and Academy of Geriatric Physical Therapy. The first purpose of this study was to explore the utilization of outcome measures that assess balance and fall risk in patients who are adults 65 years and older within the outpatient physical therapy setting. Secondary purposes included determining the most frequently used outcome measures and examining associations between demographic variables and clinicians' screening habits.

Corresponding Author a: Texas State University, Round Rock, Texas, USA.e-mail:dm78@txstate.edu

Author $\sigma$ : Texas State University, Round Rock, Texas, USA.
Methods and Measures: This study consisted of a confidential short survey distributed from November 2019 to April 2020 using Qualtrics Survey Software. A convenience sample of 205 licensed physical therapists (PTs) $(n=185)$ and physical therapist assistants (PTAs) $(n=20)$ who practice in an outpatient physical therapy setting and treat older adults in Texas. Participants identified through a Texas Physical Therapy Association database of licensed PTs and PTAs completed the survey with questions regarding demographic data, physical therapy practice, and utilization of outcome measures for balance and fall risk. Frequency distributions were generated to illustrate demographic variables and $\mathrm{Chi}$ square analyses $(p=0.05)$ were used to explore associations between demographic variables and utilization of the outcome measures.

Results: Most respondents (66.83\%) reported screening all older adults 65 years and older for balance and fall risk, regardless of diagnosis or condition. A significant positive relationship was found between the Years of Practice category and whether or not patients were screened and between the Percentage of Elders Treated in Practice categories and whether or not a clinician screened all elders over 65 for fall risk. The outcome measures most frequently utilized were the Timed Up and Go (86.57\%), Berg Balance Scale (68.16\%), and 5 Time Sit to Stand (64.18\%).

Conclusion: This study indicated that nearly $1 / 3(33.17 \%)$ of practicing PTs and PTAs in the outpatient physical therapy setting are not assessing adults 65 years and over for balance dysfunction and fall risk despite current recommendations, thus potentially neglecting to identify at-risk patients. The 3 diagnostic tests and measures most often utilized to assess fall risk were the Timed Up \& Go (86.57\%), Berg Balance Scale (68.16\%), and the 5-times sit-to-stand (64.18\%).

Clinical Relevance: Results may assist practicing PTs and PTAs with recognizing that it is essential that utilization of balance and fall risk outcome measures are incorporated into clinicians' practice to improve the standard of care when treating adults 65 years and older. Furthermore, this study may guide PTs and PTAs with the selection of continuing education that addresses the appropriate outcome measures that are recommended for evidenced-based practice.

Keywords: older adults, falls, balance, outcome measures, screening, physical therapy practice. 


\section{INTRODUCTION}

T he rising occurrence of falls in older adults is a major public health concern due to its impact on health outcomes, quality of life, and treatment costs. ${ }^{1-5}$ Proper screening and assessment by physical therapy practitioners with the implementation of appropriate outcome measures to identify at-risk patients can greatly reduce the occurrence of falls in older adults. This study was designed to explore physical therapists' and physical therapist assistants' utilization of evidence-based outcome measures to screen and assess for balance and fall risk in adults 65 years and up.

\section{BACKGROUND}

Nearly $1 / 3$ of all adults 65 years and older fall annually secondary to numerous intrinsic and extrinsic factors, including neuromuscular impairments, functional mobility deficits, lower extremity muscle weakness, medication side effects, and environmental obstacles. ${ }^{1-5}$ This subsequently leads to an increased risk of recurrent falls by 50 percent. Falling incidents are the leading cause of unintentional death and are the most common mechanism for fractures and traumatic brain injuries for patients 65 years and older ${ }^{6-8}$ Previous literature has established that well-designed exercise interventions significantly prevent falls in the general older adult population with some studies indicating a $30 \%$ decrease in falls in the group receiving exercise intervention compared to the control group. 9${ }^{12}$ Decreasing falls to this extent results in a potential decrease in healthcare expenditure by 94 to 422 million dollars annually. ${ }^{13}$ Additionally, falls are not only responsible for exponential healthcare costs and increased morbidity and mortality rates, but they also lead to decreased community mobility, a fear of falling, and an increased likelihood of early admission to longterm care facilities or nursing homes. ${ }^{14}$

Screening, assessment, and management of patients who may be at a risk of falling is within the scope of physical therapy practice and is recommended to be implemented as part of the plan of care in order to improve overall health outcomes for the geriatric population. ${ }^{15}$ Numerous representatives for this population, including the American Geriatric Society (AGS), American Physical Therapy Association (APTA), Academy of Neurologic Physical Therapy (ANPT), Academy of Geriatric Physical Therapy (AGPT), and the Centers for Disease Control and Prevention (CDC), state that all individuals over the age of 65 years should be screened for risk of falling, regardless of their condition or diagnosis. Several of these institutions have also released best practice recommendations or clinical prediction guidelines designed to assist healthcare individuals during the screening and assessment process, which include the implementation of outcome measures targeted for balance and fall risk. 4,10,14-17

A significant number of fall risk assessment tools have been identified as having good reliability and good validity for identifying fall potential with communitydwelling older adults. Park (2018) performed a systemic review and meta-analysis on diagnostic studies through June 2016 to assess which of these tools best predict the risk of falls in the elderly. Results suggested that two assessment tools used in combination as opposed to a single assessment tool maximizes the characteristics and predictability of each test. Furthermore, it was concluded that for assessing the risk of falls among community-dwelling older adults, the Timed Up and Go (TUG) test and the Berg Balance Scale (BBS) should be used in combination to increase diagnostic accuracy of the older adult's fall risk. ${ }^{18}$ Lusardi, et al (2017) also performed a systematic review and meta-analysis using posttest probability of the existing literature to assist clinicians with selecting the best diagnostic tool(s) to examine an older adult's risk of falling. They concluded that the BBS, TUG, and the 5-times sit-to-stand (FTSTS) test are currently the most evidence-supported functional measures to determine the risk of future falls for community-dwelling older adults. ${ }^{19}$

Research has previously established that physical therapist or physical therapist assistant utilization of outcome measures to screen and provide a multifactorial assessment of balance and fall risk in patients 65 years and older is an essential aspect of their rehabilitation. This practice aids in the reduction of falls, fall-related injuries, fall-related deaths, and healthcare expenses, while promoting patient quality of life, community mobility, and social participation. However, more research is needed to determine whether these measures are consistently being utilized in outpatient physical therapy practice.

Therefore, the primary purpose of this study was to explore the utilization of outcome measures to assess balance and fall risk in patients 65 years and up within the outpatient physical therapy setting. Secondary aims included determining the most frequently used outcome measures as well as association between clinician demographic variables and screening habits. Our primary research question was: Is there sufficient utilization of standardized outcome measures to assess balance and fall risk in geriatric patients treated in the outpatient physical therapy setting? Our second research question was: Are common practice, standardized outcome measures utilized by PTs and PTAs in outpatient settings the same tools recommended by current research evidence?

\section{ili. Methods}

The study was conducted from November 2019 to April 2020 by four Doctor of Physical Therapy (DPT) 
students at Texas State University. Participants were recruited by means of non-probability, convenience sampling through a physical therapist and physical therapist assistant licensure database provided by the Texas Physical Therapist Association. All participants were sent a survey which included informed consent prior to participation and informed about their right to withdraw at any point during the survey process. Inclusion criteria required that participants needed to be a licensed, practicing physical therapist or physical therapist assistant, practice in an outpatient physical therapy setting, and be involved in the treatment of patients aged 65 years or older. This study was approved by the Institutional Review Board (IRB) of Texas State University, San Marcos, Texas on August 30, 2019.

Qualtrics Survey Software was used for survey distribution and collection of participant responses. This system requires a two-step login process to further ensure participant confidentiality. The 23-item survey consisted of closed-ended multiple-choice questions with options to provide additional open-ended responses on select questions to gain as much pertinent information as possible. The survey also provided outcome measures as answer choices for clinical utilization based on recommendations regarding screening and assessing balance in those 65 years and older from several organizations, including the APTA, ANPT, AGPT, AGS, and the CDC. ${ }^{6,14-18}$ In addition, the survey also included items regarding level of education, APTA sections, and postgraduate training, such as continuing education, board certifications, residency training, or fellowships, developed based on information retrieved from the American Board of Physical Therapy Residency and Fellowship Education (ABPTRFE) website. $^{19-20}$ Demographic data was included in the survey, however no personal identifiable information was collected. All responses were recorded anonymously and confidentiality was maintained according to the IRB protocol.

\section{a) Data Analysis}

All survey responses were either nominal or ordinal data measurement levels therefore nonparametric statistical analysis methods were conducted using SPSS (IBM vs. 26). Statistical analysis included Pearson's Chi Square frequency contingency tables to explore demographic and clinic data in terms of descriptive trends and bivariate relationships with an alpha level set at $p=0.05$.

\section{Results}

A total of 574 survey responses were recorded, with 205 participants meeting the inclusion criteria for this study. Demographic data was collected for each member, which consisted of age, gender, professional license, level of education, state of education, state of practice, number of years in practice, and APTA membership status (Figures 1, 2). Of the 205 responses, $185(90.24 \%)$ were licensed physical therapists and 20 $(9.76 \%)$ were licensed physical therapist assistants. The majority of the participants received their education (65.69\%) and practiced (93.17\%) in the state of Texas. Participants were asked to report their entry-level physical therapy degree as well as any additional PT degrees earned (Figure3).Out of the participants surveyed, $21.95 \%$ were board certified specialists in at least one area, $7.32 \%$ completed residency programs, and $3.9 \%$ completed fellowship programs.

Of the 205 participants, $51.71 \%$ reported that at least $50 \%$ of their caseloads involved patients who are 65 years or older. Only $66.83 \%$ of participants reported that they screen balance and fall risk on all patients age 65 and older, regardless of their diagnosis or condition. When questioned what percentage of $65+$ year old patients are treated for balance and fall risk, regardless of their diagnosis or condition, 2 respondents stated $0 \%$, 52 stated 1-25\%, 63 stated 25-50\%, 54 stated 51-75\%, and 34 stated $75-100 \%$ (Figures 4-6)

Participants were prompted to select all balance outcome measures that they regularly utilized in practice for screening and assessing balance. Of those selected, the Timed Up and Go (TUG), Berg Balance Scale (BBS), and the 5-Time Sit to Stand (FTSTS) were the three most frequently utilized measures, with rates of $86.57 \%$, $68.16 \%$, and $64.18 \%$, respectively. When asked to select the top three most utilized outcome measures, the top responses remained the TUG (145/197 respondents), BBS (102/197 respondents), and FTSTS (101/197 respondents). (Tables 1,2)

Results from Pearson's Chi Square analysis indicated that there was no significant association between whether clinicians screened patients for balance and fall risk regardless of diagnosis or condition and APTA membership status $(p=0.137)$, age groups $(p=0.152)$, highest physical therapy degree earned $(p=0.432)$, or whether the clinician was a PT or PTA $(p=0.237)$. However, there was a significant positive relationship found between years of practice category and whether or not patients were screened for balance and fall risk regardless of diagnosis or condition $\left(\mathrm{X}^{2}=\right.$ 24.70, $p=0.018$ ).

There was also a significant predictive relationship between the Percentage of Elders Treated in Practice categories and whether or not a clinician screened all elders over 65 for balance problems $\left(\mathrm{X}^{2}=\right.$ 34.551, $p<0.001$ ) with a large effect size as indicated by the Phi Coefficient $(\Phi=0.411, p<0.001)$.

It must also be noted that $67.8 \%$ of respondents in our sample were biased towards younger ages or between 20 and 50 years of age categories $\left(\mathrm{X}^{2}=\right.$ 95.029, $p<0.001$ ). In addition, a significant proportion of our sample or $48.8 \%$ were in practice less than 15 years (Categories: $11-15,6-10,1-5$ and $<1$ years) $\left(\mathrm{X}^{2}=\right.$ 
72.122, $\mathrm{p}<0.001)$. Other findings indicated a significant bias to one primary area of practice, Orthopaedics $\left(X^{2}=557.015, p<0.001\right)$. What was surprising is that a significant number of respondents $(62 \%)$ were not members of the APTA $\left(X^{2}=3.352, p=\right.$ 0.001). Finally, it was good to note that results indicated that a significant number of clinicians (65.8\%) did screen elders 65 and older for balance and fall risks $\left(X^{2}=4.749\right.$, $\mathrm{p}<0.001)$.

\section{Discussion}

The findings of this study indicate that while many outpatient physical therapists are utilizing outcome measures to screen or assess balance and fall risk in patients $65+$ years, nearly $1 / 3$ of clinicians do not. Additional trends as presented in this study indicate that the most widely utilized outcome measures for assessing balance are the BBS,TUG and FTSTS though there are many other valid, reliable, and recommended outcome measures also being implemented. Utilization of the aforementioned outcome measures is consistent with recommendations regarding best practice and may contribute to an enhanced overall plan of care for this patient population. ${ }^{15}$

Findings about trends with high percent use of BBS, TUG and FTSTS aligned with findings of Park (2018) who indicated evidence-based value of combination of tests. ${ }^{21}$ The trends from this study also agree with Lusardi, et al (2017) who concluded that these 3 diagnostic tools are currently the most evidencesupported functional measures to determine the risk of future falls for community-dwelling adults. ${ }^{19}$ Additionally, clinician years' experience and practice appear to influence whether or not patients 65 and over were screened for falls. This is in agreement with Stroud's study that included 316 physical therapist respondents. ${ }^{23}$

APTA membership did not appear to influence whether or not screening took place however results may have been biased due to low proportion of memberships in general. Previous evidence is conflicting with Stroud (2014) having a positive association with APTA membership and the utilization of outcome measures, yet Anderson et. al. (2015) found outcome measures utilization was not significantly correlated with APTA membership. ${ }^{23,24}$

The lack of implementation of these screening tools into practice could potentially lead to an increased occurrence of falls in this patient population, thus resulting in increased healthcare expenditure, morbidity, and mortality rates, and decreased independence and quality of life.

\section{Clinical Implications}

This study raises consequential clinical implications for outpatient physical therapists and physical therapist assistants. To assist in the prevention of falls, it is essential that all outpatient physical therapy practitioners incorporate a component of screening and assessing balance and fall risk for all patients $65+$ years old into their clinical practice. These clinicians have the potential to make a significant impact on numerous personal facets for this patient population, as well as on global and national healthcare expenditure. PTs and PTAs have the responsibility for continued education and maintenance of knowledge regarding best clinical practice guidelines concerning screening and multifactorial assessments for balance of fall risk in order to improve the standard of care for the geriatric patient population.

The use of these tools will also become increasingly important as reimbursement models shift from quantity-based care to quality-based care, as they provide quantitative data on a patient's progression throughout the course of treatment, which directly translates to justification of services. ${ }^{18}$ With Medicare's recent implementation of the Quality Payment Program (QPP), Merit-Based Incentive Payment Systems (MIPS) will become one of the focal means of justifying treatment to obtain reimbursement of services. This system utilizes outcome measures, specifically those relating to fall risk, to illustrate medical necessity and patient progress, which then determines reimbursement rates. Therefore, Medicare reimbursement rates are directly related to participation in MIPS and the utilization of outcome measures, thus reinforcing the importance of adopting regular screening protocols into clinicians' physical therapy practice. ${ }^{25,26}$

\section{Limitations}

This study is limited in terms of generalizability as the sample was biased largely towards clinicians practicing in Texas secondary to the convenience sampling method utilized. Recent graduates with physical therapy degrees were also largely underrepresented. In addition, this study was limited to the outpatient setting only, which does not account for the utilization of these tools in other practice environments, such as skilled nursing facilities and hospital-based, inpatient rehabilitation units. Additionally, the survey was only offered in English, thus potentially eliminating participants due to a language barrier. Finally, statistical analysis methods used only non-parametric statistics. Future studies should consider more parametric measures to support multivariate regression prediction models about population trends.

\section{Vi. Conclusion}

Incorporation of outcome measures to screen and assess balance and fall risk for all patients 65 years or older, regardless of their diagnosis or condition, is vital for the prevention of falls in this patient population. Despite numerous professional organizations' 
recommendations to screen balance and fall risk in all patients 65 years and older, this study shows that only $2 / 3$ of outpatient physical therapists are doing so. The failure to incorporate this into practice could potentially result in practitioners neglecting to identify a significant portion of the population that may be at risk for falls. Further research should be conducted to encompass a broader sample of outpatient physical therapists and physical therapist assistants to determine the incorporation of this practice in other regions across the United States. Moreover, potential future research to discern the clinical rationale for outcome measure selection would also be beneficial for the profession of physical therapy regarding the rehabilitation of the geriatric population.

\section{References Références Referencias}

1. Bjerk $M$, Brovold $T$, Skelton $D$, Liu-Ambrose $T$, Bergland A. (2019). Effects of a falls prevention exercise programme on health-related quality of life in older home care recipients: a randomised controlled trial, Age and Ageing,48(2):213-219, https://doi.org/10.1093/ageing/afy192

2. Bjerk M, Brovold T, Skelton D, Bergland A.(2017) A falls prevention programme to improve quality of life, physical function and falls efficacy in older people receiving home help services: study protocol for a randomised controlled trial. BMS Health Serv Res. 17:559. https://doi: 10.1186/s12913-017-2516-5

3. Haddad YK,Bergen G, Curtis S. (2019) Estimating the Economic Burden Related to Older Adult Falls by State, Journal of Public Health Management and Practice, 25(2): E17-E24. https://doi: 10.1097/ $\mathrm{PHH} .0000000000000816$

4. Stevens J \& Lee R. (2018) The Potential to Reduce Falls and Avert Costs by Clinically Managing Fall Risk. American Journal of Preventive Medicine, 55(3):290-297. https://doi.org/10.1016/j.amepre. 2018.04.035

5. Bergen G, Stevens M, Kakara R, Burns E. (2019) Understanding Modifiable and Unmodifiable Older Adult Fall Risk Factors to Create Effective Prevention Strategies. American Journal of Lifestyle Medicine. https://doi.org/10.1016/j.amepre.2018.04.035

6. Center for Disease Control and Prevention website. Older adult falls. https://www.cdc.gov/homeand recreationalsafety/falls/adultfalls.html. Accessed July 22, 2020

7. Burns \& Kakara R. (2018) Deaths from Falls Among Persons Aged $\geq 65$ Years - United States, 20072016. Morbidity and Mortality Weekly Report (CDC). 67(18): 509-514. https://doi: 10.15585/mmwr. mm6718a1

8. Taylor C, Bell J, Breiding M, Xu L. (2017) Traumatic Brain Injury-Related Emergency Department Visits, Hospitalizations, and Deaths - United States, 2007-
2013. MMWR Surveill Summ.66(9):1-16. https://doi: 10.15585/mmwr.ss6609a1

9. Sherrington C \& Tiedemann A. (2015) Physiotherapy in the prevention of falls in older people. Journal of Physiotherapy, 61(2):54-60. https://doi.org/10.1016/ j.jphys.2015.02.011

10. Fairhall N, et al. (2014) Effect of a multifactorial, interdisciplinary intervention on risk factors for falls and fall rate in frail older people: a randomised controlled trial. Age Ageing, :43(5):616-622

11. Lee SH\& Yu S. (2020) Effectiveness of multifactorial interventions in preventing falls among older adults in the community: A systematic review and metaanalysis. Int $\mathrm{J}$ Nurs Stud. 106:103564. https://doi:10.1016/j.ijnurstu.2020.103564

12. Guirguis-Blake JM, Michael YL, Perdue LA, Coppola EL, Beil TL Thompson JH. (2018) Interventions to Prevent Falls in Community-Dwelling Older Adults: A Systematic Review for the U.S. Preventive Services Task Force [Internet]. Rockville (MD): Agency for Healthcare Research and Quality (US) (Evidence Synthesis, Nol.159.) https://www.ncbi.nlm.nih.gov/ books/NBK525700/

13. Florence C, Bergen G, Atherly A, Burns E, Stevens J, Drake C. (2018) Medical costs of fatal and nonfatal falls in older adults. J Am Geriatr Soc. 266(4): 693-698. https://doi: 10.1111/jgs.15304

14. American Geriatrics Society. Summary of the Updated American Geriatrics Society/British Geriatrics Society Clinical Practice Guideline for Prevention of Falls in Older Persons. J Am Geriatr Soc. 2011;59(1):148-157

15. American Physical Therapy Association website. Balance and Falls. https://www.apta.org/ BalanceFalls/. Accessed July 22, 2020

16. Avin KG, et al. (2015) Management of falls in community-dwelling older adults: clinical guidance statement from the Academy of Geriatric Physical Therapy of the American Physical Therapy Association. Phys Ther. 95(6):815-834.

17. Moore JL, et al. (2018) A core set of outcome measures for adults with neurologic conditions undergoing rehabilitation: a clinical practice guideline. J Neurol Phys Ther. 42(3):174-220.

18. Centers for Medicare and Medicaid Services website. Quality payment program. https://qpp. cms.gov/about/qpp-overview. Accessed July 24,2020

19. American Board of Physical therapy Residency and Fellowship Education website. About Fellowships. https://www.abptrfe.org/ForParticipants/AboutFellow ships/. Accessed July 24, 2020

20. American Board of Physical Therapy Residency and Fellowship Education website. About Residencies. https://www.abptrfe.org/ForParticipants/AboutResid encies/. Accessed July 24, 2020 
21. Park SH. (2018) Tools for assessing fall risk in the elderly: a systematic review and meta-analysis. Aging Clin Exp Res. 30:1-16. https://doi:10.1007/s40520-017-0749-0

22. Lusardi M, et al. (2017) Determining Risk of Falls in Community Dwelling Older Adults: A Systematic Review and Meta-analysis Using Posttest Probability. Journal of Geriatric Physical Therapy, 40(1):1-36 doi: 10.1519/JPT.0000000000000099.

23. Stroud MA. (2014). Physical Therapists' Clinical Practices Regarding Intrinsic and Extrinsic Fall Risk Factors and Their Attitudes Toward the Use of Evidence-based Practice. [Unpublished doctorate dissertation]. Southern Illinois University Carbondale.

24. Anderson HD \& Sullivan JE. (2015). Outcome Measures for Persons with Acute Stroke: A Survey of Physical Therapists' Practice in Acute Care and
Acute Rehabilitation Settings. Journal of Acute Care Physical Therapy. https//:doi:10.1097/JAT. 0000000000000031

25. Smith H. (April, 2019). American Physical Therapy Association website. MIPS Is Here. https://www. apta.org/apta-magazine/2019/04/01/compliancematters-mips-is-here. Accessed August 10, 2020

26. American Physical Therapy Association website. Merit-Based Incentive Program. https://www.apta. org/your-practice/payment/value-based-paymentmodels/quality-payment-program/merit-basedincentive-program. Accessed August 10, 2020

Acknowledgement of any financial support: None to report

Conflicts of Interest:

None to report

Abbreviations Used in Article

\begin{tabular}{|l|l|}
\hline \multicolumn{1}{|c|}{ Abbreviations } & \multicolumn{1}{c|}{ Definitions } \\
\hline ABPTRFE & $\begin{array}{l}\text { American Board of Physical Therapy Residency } \\
\text { and Fellowship Education }\end{array}$ \\
\hline AGPT & Academy of Geriatric Physical Therapy \\
\hline AGS & American Geriatric Society \\
\hline ANPT & Academy of Neurologic Physical Therapy \\
\hline APTA & American Physical Therapy Association \\
\hline BBS & Berg Balance Scale \\
\hline CDC & Centers for Disease Control and Prevention \\
\hline FTSTS & 5-Times Sit-to-Stand \\
\hline IRB & Institutional Review Board \\
\hline MIPS & Merit-Based Incentive Payment Systems \\
\hline PTAs & Physical Therapist Assistants \\
\hline PTs & Physical Therapists \\
\hline QPP & Quality Payment System \\
\hline TUG & Timed Up and Go \\
\hline
\end{tabular}




\section{How many years have you been practicing as a PT or PTA?}

50

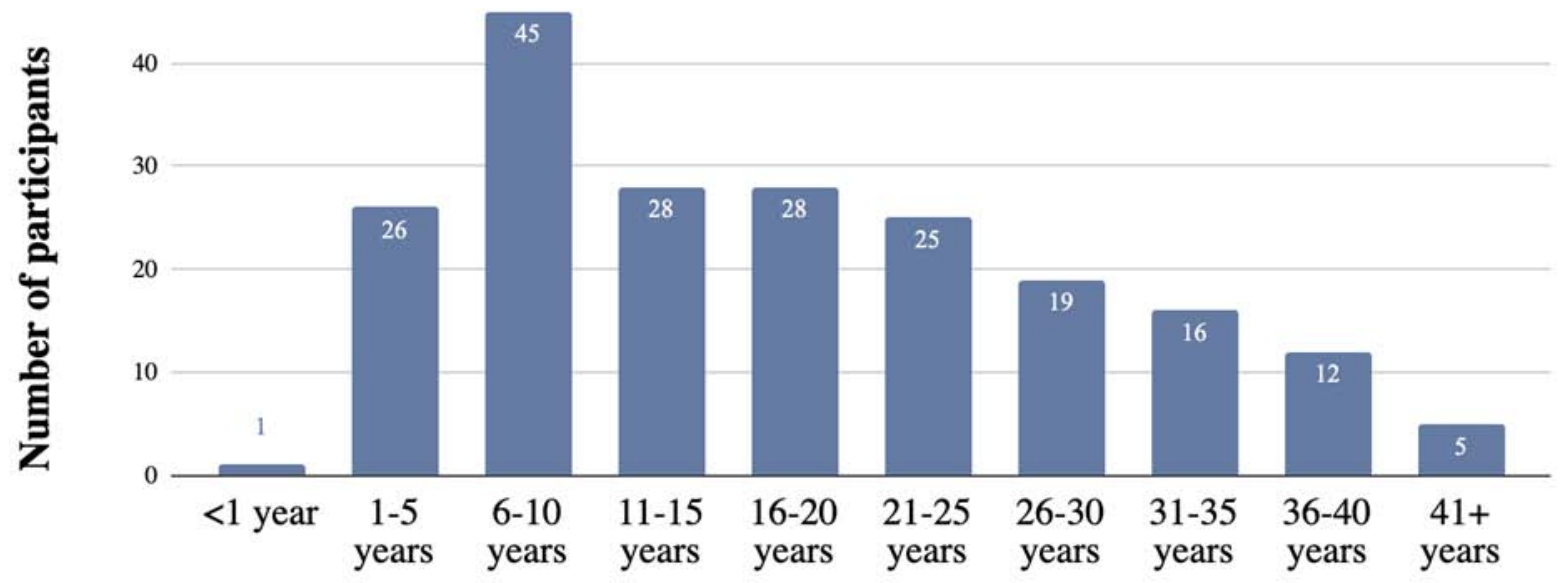

Number of Years Practicing

Figure 1: Number of Years Practicing

Are you currently a member of the American Physical Therapy Association?

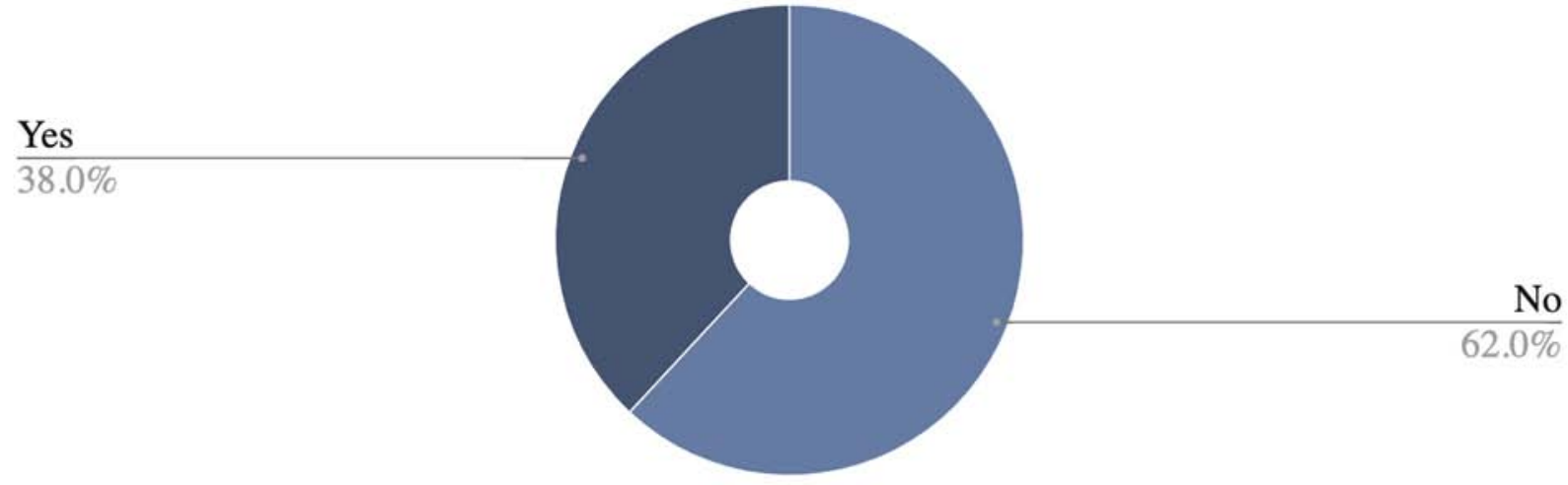

Figure 2: APTA Membership 
What entry level academic physical therapy degree did you earn?

80

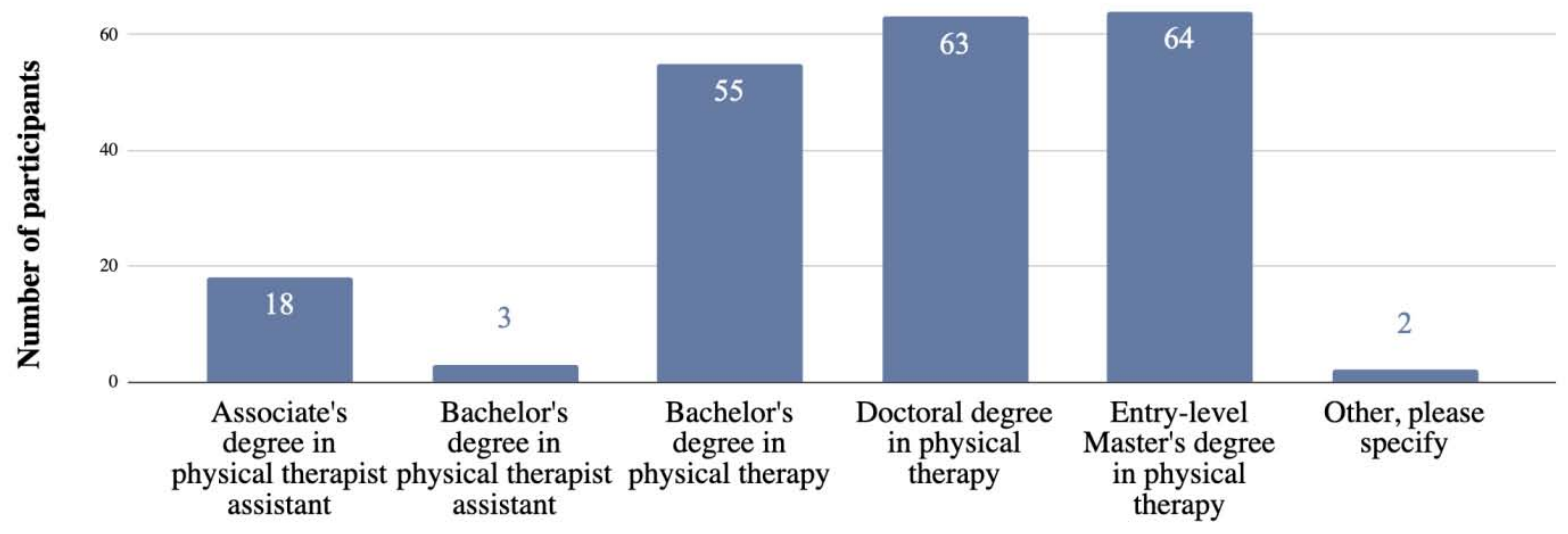

Entry-Level Physical Therapy Degree Earned

Figure 3: Entry - Level Physical Therapy Degree

\section{What percentage of the patients you treat are 65 years or older?}

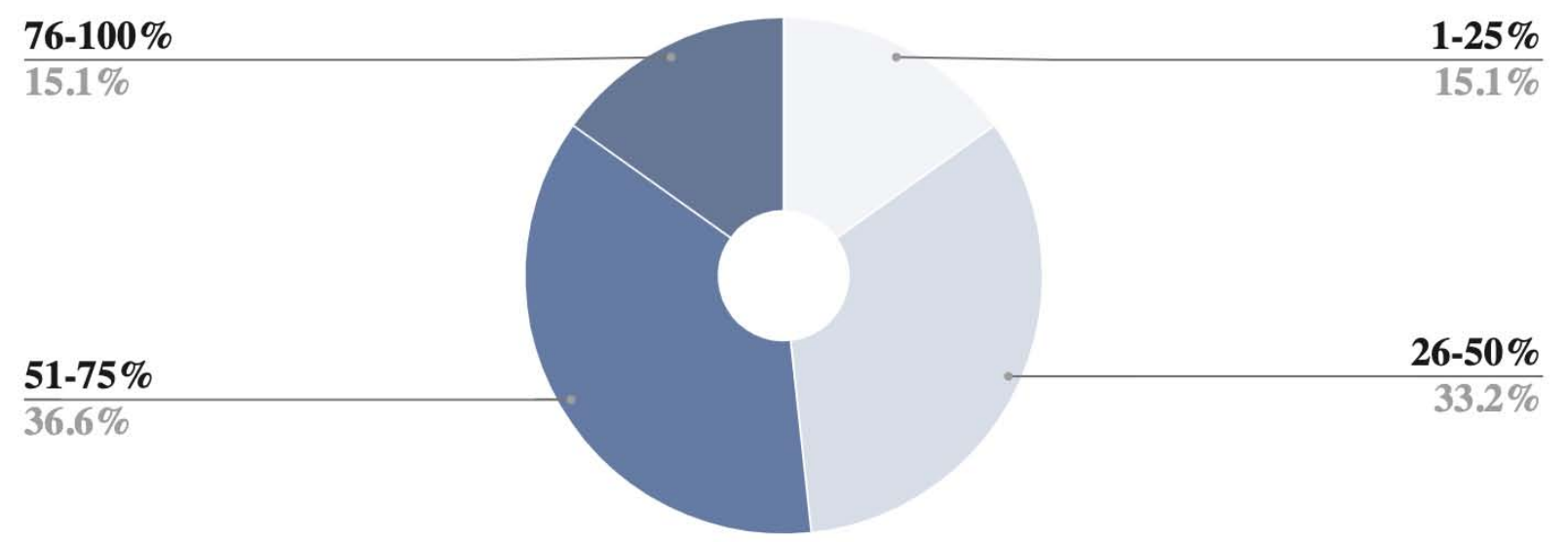

Figure 4: Caseload Age 
Do you screen balance/fall risk on ALL patients 65 years or older, regardless of their diagnosis or condition?



Figure 5: Screen Balance/Fall Risk

What percentage of the patients who are $65+$ years are treated for balance or fall risk in your clinic, regardless of diagnosis or condition?

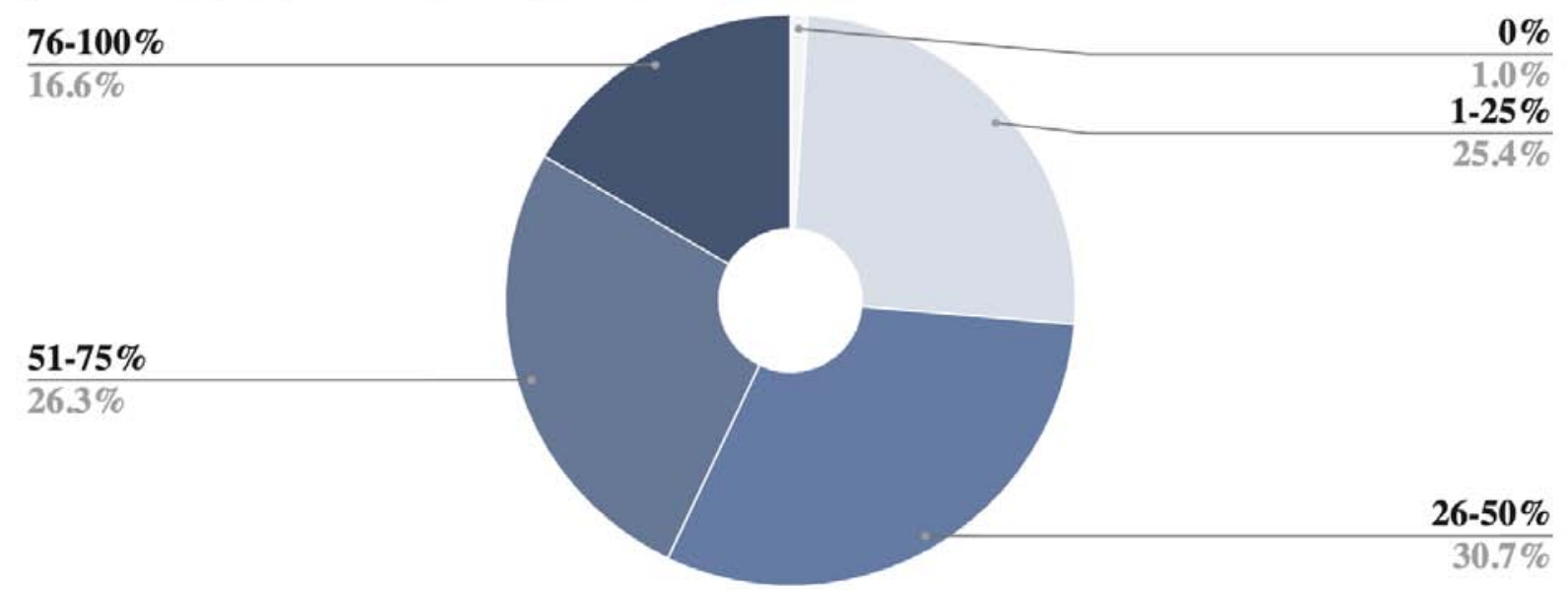

Figure 6: Treat Balance/Fall Risk

Table 1: All Utilized Balance Outcome Measures

\begin{tabular}{|c|c|c|}
\hline Q22 Which outcome measures do you regularly utilize in your practice? & Count & Percent of $n$ \\
\hline Activities Specific Balance Confidence Scale (ABC) & 57 & $28.36 \%$ \\
\hline Balance Evaluation Systems Test (BEST) & $4.98 \%$ \\
\hline Berg Balance Scale (BBS) & 10 & $68.16 \%$ \\
\hline Clinical Test of Sensory Integration of Balance (CTSIB) & 137 & $19.40 \%$ \\
\hline Dynamic Gait Index (DGI) & 39 & $44.78 \%$ \\
\hline 5 Time Sit to Stand (FSTS) & 90 & $64.18 \%$ \\
\hline Fullerton Advanced Balance Scale (FAB) & 129 & $1.49 \%$ \\
\hline Functional Reach Test & 3 & $19.90 \%$ \\
\hline Functional Gait Assessment (FGA) & 40 & $22.39 \%$ \\
\hline MiniBEST & 45 & 27 \\
\hline Performance Oriented Mobility Assessment (POMA) & 8 & $3.98 \%$ \\
\hline
\end{tabular}




\begin{tabular}{|c|c|c|}
\hline 10 Meter Walk Test (10MWT) & 39 & $19.40 \%$ \\
\hline Timed Up and Go (TUG) & 174 & $86.57 \%$ \\
\hline Other, please specify & 48 & $23.88 \%$ \\
\hline Grand Total of Responses & 846 & \\
\hline Total Number of Participants $(n)$ & 201 \\
\hline
\end{tabular}

Table 2: Top Three Utilized Balance Outcome Measures

\begin{tabular}{|c|c|c|}
\hline $\begin{array}{c}\text { Q23 Of the outcome measures that you selected in the previous } \\
\text { question, please select the three measures that you most frequently } \\
\text { utilize clinically. }\end{array}$ & Count & Percent of $n$ \\
\hline Activities Specific Balance Confidence Scale (ABC) & 29 & $14.72 \%$ \\
\hline Balance Evaluation Systems Test (BEST) & 3 & $1.52 \%$ \\
\hline Berg Balance Scale (BBS) & 102 & $51.78 \%$ \\
\hline Clinical Test of Sensory Integration of Balance (CTSIB) & 16 & $8.12 \%$ \\
\hline Dynamic Gait Index (DGI) & 47 & $23.86 \%$ \\
\hline 5 Time Sit to Stand (FSTS) & 101 & $51.27 \%$ \\
\hline Fullerton Advanced Balance Scale (FAB) & 0 & $0.00 \%$ \\
\hline Functional Reach Test & 7 & $3.55 \%$ \\
\hline Functional Gait Assessment (FGA) & 22 & $11.17 \%$ \\
\hline MiniBEST & 12 & $6.09 \%$ \\
\hline Performance Oriented Mobility Assessment (POMA) & 5 & $2.54 \%$ \\
\hline 10 Meter Walk Test (10MWT) & 19 & $9.64 \%$ \\
\hline Timed Up and Go (TUG) & 145 & $73.60 \%$ \\
\hline Other, please specify & 32 & $16.24 \%$ \\
\hline Grand Total of Responses & 540 & \\
\hline Total Number of Participants (n) & 197 & \\
\hline
\end{tabular}

